ORIGINAL ARTICLE

\title{
Partial rupture of the proximal Achilles tendon: a differential diagnostic problem in ultrasound imaging
}

\author{
R Kayser, K Mahlfeld, C E Heyde
}

Br J Sports Med 2005;39:838-842. doi: 10.1136/bjsm.2005.018416

See end of article for authors' affiliations

....................

Correspondence to: Ralph Kayser, Charité University Medicine Berlin, Trauma and Reconstructive Surgery, Campus Benjamin Franklin, Hindenburgdamm 30, D12200 , Berlin, Germany; ralph.kayser-berlin@ t-online.de

Accepted 12 April 2005

\begin{abstract}
Objectives: The aim of this study was to determine whether ultrasound can correctly visualise partial ruptures of the proximal Achilles tendon.

Method: This was a prospective study in which all chronic Achilles tendon injury patients seen at three centres in Germany from 1998 to 2003 were screened. All patients with clinical and/or sonographic signs of abnormalities in the region of the proximal third of the Achilles tendon and tendomuscular junction were included in the analysis. Each of these cases was evaluated by ultrasound following an assessment protocol. Patients with ambiguous ultrasound findings and/or clinical signs were additionally assessed by magnetic resonance imaging (MRI).

Results: Sonomorphologic changes suggestive of an abnormality in the proximal third of the Achilles tendon were detected in 13 out of 320 patients $(4.2 \%)$ with recurring Achilles tendon complaints. Thirteen patients had clinical signs but no sonographic changes in the tendon. The sonographic diagnosis was correct in 19 cases. In six of the 26 cases studied, MRI was needed to establish the correct diagnosis of partial intratendinous rupture of the proximal Achilles tendon. Sensitivity was 0.5 , specificity was 0.81 , and the overall agreement of the ultrasound examination was $61.5 \%$. All patients were asymptomatic at follow up at a mean of 14 months (range 12-17 months) after surgery.

Conclusions: Ultrasound is a useful tool for evaluation of proximal Achilles tendon complaints. However, ultrasound is not sufficiently reliable for diagnosis of all pathologies, especially partial ruptures of the Achilles tendon. Thus, the definitive diagnosis must be established by MRI.
\end{abstract}

hronic Achilles tendon complaints are commonly observed in top athletes in all running, jumping, and ball sports disciplines. Degenerative changes (tendinopathies), tendinitis, peritendinitis, partial or complete tendon rupture, osseous changes (calcaneal spurs, Haglund's deformity), and bursitis are common causes of chronic Achilles tendinopathies. ${ }^{12}$ Knowing the exact diagnosis is crucial for establishing an adequate treatment plan. The great value of ultrasound lies in its ability to differentially diagnose Achilles tendinopathies in athletes. ${ }^{3}$ Assessment of acute Achilles tendon ruptures at their typical site of occurrence is certainly the domain of ultrasound and an unequivocal ultrasound diagnosis is usually possible in these cases. ${ }^{1}$ Moreover, ultrasound is now used with good success to monitor the results of treatment, both conservatively and intraoperatively. ${ }^{56}$

Experimental $^{78}$ and clinical studies ${ }^{9}$ for the assessment of the various tendinopathies grouped under the heading "achillodynia" have been published. Since MRI was established as a method for evaluating musculoskeletal system in $1985,{ }^{10}$ rapid developments have occurred. ${ }^{11}$ Jacobson, ${ }^{12}$ who presented a comprehensive study comparing the efficacy of ultrasound and MRI for diagnosis of tendinopathies and musculoskeletal diseases in 1999, pointed out the difficulties in distinguishing between degenerative changes and partial ruptures of the Achilles tendon. Movin et al demonstrated in 1998 that the use of contrast medium improves MRI of Achilles tendinopathies. ${ }^{13}$ Several publications on the ultrasound diagnosis of partial ruptures of the Achilles tendon are available. ${ }^{14-16}$ When fresh, a partial rupture against the background of an antecedent tendon injury is often more difficult to visualise than a complete rupture of the Achilles tendon, but can be detected with sufficient reliability. ${ }^{913}{ }^{15}$ If the lesion is older, diagnostic imaging can be quite difficult. ${ }^{17}$ Lesions in the proximal third of the Achilles tendon are more difficult to visualise by ultrasound than those in the medial and distal parts of the tendon. The sonographic presence of microtears in the proximal third of the Achilles tendon is not uncommon, especially in serious athletes, but usually is not of primary clinical importance. ${ }^{18}$

\section{METHODS}

All patients presenting with proximal Achilles tendon complaints during the period 1998 to 2003 were screened prospectively. Their clinical status, sonomorphologic features (longitudinal and transverse tendon diameter, thickness of the peritendineum, calcification, accumulation of fluid, tendon continuity), clinical findings (swelling, bruising, tenderness, signs of rupture, site of pain), and history data (trauma, duration and characteristics of pain, sports history) were evaluated.

\section{Ultrasound procedure}

A 7.5 MHz linear transducer was used to generate images of the Achilles tendon in horizontal and transverse planes on both the affected and contralateral side. Adara and Sonoline Prima SLC devices were used (Siemens, Erlangen, Germany). A $10 \mathrm{MHz}$ linear transducer was also available for additional studies as needed. Each patient was examined prone, with the feet in a neutral position, followed by a dynamic ultrasound examination.

\section{MRI procedure}

The 13 patients with ultrasound findings suggestive of proximal tendon injuries were additionally assessed by MRI. MRI images were obtained using T1 and T2 weighted sequences taken at ultrafast spin echo frequencies following intravenous contrast administration. 
Table 1 Number of cases with sonographic abnormalities in the proximal Achilles tendon

\begin{tabular}{ll}
\hline Centre & Number of cases \\
\hline Berlin & $5(2)$ \\
Magdeburg & $5(1)$ \\
Bad Berka & $3(2)$ \\
\hline $\begin{array}{l}\text { The number of partial ruptures are given in parentheses. Total number of } \\
\text { cases: } n=13(5) .\end{array}$
\end{tabular}

\section{RESULTS}

Out of a total population of 320 patients, sonomorphologic changes suggestive of proximal Achilles tendon disorders were detected in 13 cases $(4.2 \%)$ (table 1 ). Thirteen $(4.2 \%)$ patients had clinical but no sonographic signs of a proximal tendon pathology.

Based on the ultrasound findings, 11 of the 26 cases were diagnosed as tendinitis and/or peritendinitis (table 2A) and one as a partial rupture. No complete Achilles tendon rupture was diagnosed by ultrasound. MRI confirmed tendinitis and/ or peritendinitis as the sole pathology in seven of 26 cases, but revealed a partial rupture of the proximal Achilles tendon in five cases (table 2B).

The sensitivity of the ultrasound examination in cases of proximal pathology was 0.5 , specificity was 0.81 , and the overall agreement of the ultrasound examination was $61.5 \%$. The positive and negative predictive numbers were 0.67 and 0.68 , respectively. MRI confirmed that the partial rupture tentatively diagnosed by ultrasound was indeed an extensive partial rupture of the proximal Achilles tendon. The sonographic findings in these five cases are summarised in table 3.

Four of the five patients with a partial rupture of the proximal Achilles tendon were high performance athletes (sprinters). One was a recreational athlete with a very intense training schedule (club level soccer practice five times a week plus matches). He stated that he had received peritendinous cortisone injections twice in the past. All these patients had a history of Achilles tendinopathies that had been treated a mean of 9 (range 3-16) months previously. The exact diagnoses at that time could not be determined, but none of the patients had had a complete rupture. All the previous tendon disorders had been treated conservatively. Afterwards, the patients had been completely asymptomatic, even under a full training load and during active competition. All reportedly had suffered a recent minor injury that had not led to any typical rupture related symptoms. Instead, they had experienced a sudden, intense pain that quickly subsided. The symptoms later increased continuously. The patients eventually became unable to play sports, but there were none of the typical signs of pseudoparesis. Full dorsal extension of the upper ankle was still possible but painful.
Table 2 Distribution of sonographic and MRI diagnoses

\begin{tabular}{lcc}
\hline Diagnosis & Number of cases & Incidence (\%) \\
\hline A. Sonographic & & \\
$\quad$ Tendinitis/tendinosis* & 7 & 27 \\
$\quad$ Peritendinitis* & 4 & 15.4 \\
$\quad$ Partial rupture & 1 & 3.8 \\
$\quad$ No abnormalities & 13 & 50 \\
B. MRI & & 15.4 \\
$\quad$ Tendinitis/tendinosis * & 4 & 11.5 \\
$\quad$ Peritendinitis* & 3 & 19.2 \\
$\quad$ Partial rupture & 5 & 50 \\
$\quad$ No abnormalities & 13 & \\
\hline *Main diagnosis. & & \\
$\mathrm{n}=26$ patients with proximal & Achilles tendon symptoms.
\end{tabular}

None of the patients had observed any characteristic signs of bruising or had heard an audible tearing of the tendon.

In clinical examination, no dent in the tendon could be palpated. However, a point of maximum pain could be located in the region of the tendon-muscle junction. This was confirmed by applying pressure with the transducer during the ultrasound examination. The point of maximum pain was determined to lie at the site of the visualised sonomorphologic changes. The complaints persisted in spite of conservative treatment. In each case, the former complaints had occurred a mean of 15 months previously.

A prominent bump on the tendon (Haglund's deformity) with changes in the typical herringbone echo pattern and an increase in tendon diameter were the most frequent findings of the static ultrasound examination (table 3). No complete break in tendon continuity could be detected, even in the forced dynamic assessment. Hypoechoic changes in echo texture within the tendon were detected in three of five cases. A partial break in tendon continuity was detected during the forced dynamic ultrasound assessment in one case. This tendon was additionally scanned with a $10 \mathrm{MHz}$ transducer, resulting in the tentative diagnosis of a partial rupture (fig 1A,B). In each case, a narrow, hypoechoic halo, both around and within the tendon, was detected in the proximal third of the Achilles tendon (fig 2). This sign, which was visualised in both planes, was limited to the proximal tendon region (table 3 ). The site of this anomaly corresponded to the point of maximum pain.

In the MRI evaluation, the Tl weighted sequences revealed signal changes commensurate with the duration of symptoms: these were chronic changes with a corresponding thickening of the tendon. The T2 weighted sequences showed a zone of higher signal intensity within the intact contours of the tendon (fig 3). Based on its distribution pattern, this was interpreted as fluid that had accumulated after a partial rupture. The one partial rupture already detected by

Table 3 Sonomorphologic features in patients with partial ruptures in the proximal third of the Achilles tendon

\begin{tabular}{|c|c|c|c|c|c|}
\hline Sonomorphologic feature & Case 1 & Case 2 & Case 3 & Case 4 & Case 5 \\
\hline $\begin{array}{l}\text { Tendon continuity } \\
\text { Change in intratendinous echo texture with echo-free zones } \\
\text { Haglund's deformity } \\
\text { Internal structure } \\
\text { Bursitis } \\
\text { Calcification } \\
\text { Peritendineum } \\
\text { Peritendinous fluid } \\
\text { Maximum tendon diameter in transverse plane }\end{array}$ & $\begin{array}{l}\text { Preserved } \\
\text { No } \\
\text { Yes } \\
\text { Changed } \\
\text { No } \\
\text { No } \\
\text { Changed } \\
\text { Yes } \\
7.4 \mathrm{~mm}\end{array}$ & $\begin{array}{l}\text { Preserved } \\
\text { Yes } \\
\text { Yes } \\
\text { Changed } \\
\text { No } \\
\text { No } \\
\text { Normal } \\
\text { Yes } \\
8.2 \mathrm{~mm}\end{array}$ & $\begin{array}{l}\text { Preserved } \\
\text { Yes } \\
\text { Yes } \\
\text { Changed } \\
\text { Yes } \\
\text { No } \\
\text { Normal } \\
\text { Yes } \\
8.4 \mathrm{~mm}\end{array}$ & $\begin{array}{l}\text { Preserved } \\
\text { No } \\
\text { No } \\
\text { Changed } \\
\text { No } \\
\text { Yes } \\
\text { Changed } \\
\text { Yes } \\
8.6 \mathrm{~mm}\end{array}$ & $\begin{array}{l}\text { Incomplete break } \\
\text { Yes } \\
\text { Yes } \\
\text { Changed } \\
\text { No } \\
\text { No } \\
\text { Changed } \\
\text { Yes } \\
7.4 \mathrm{~mm}\end{array}$ \\
\hline
\end{tabular}



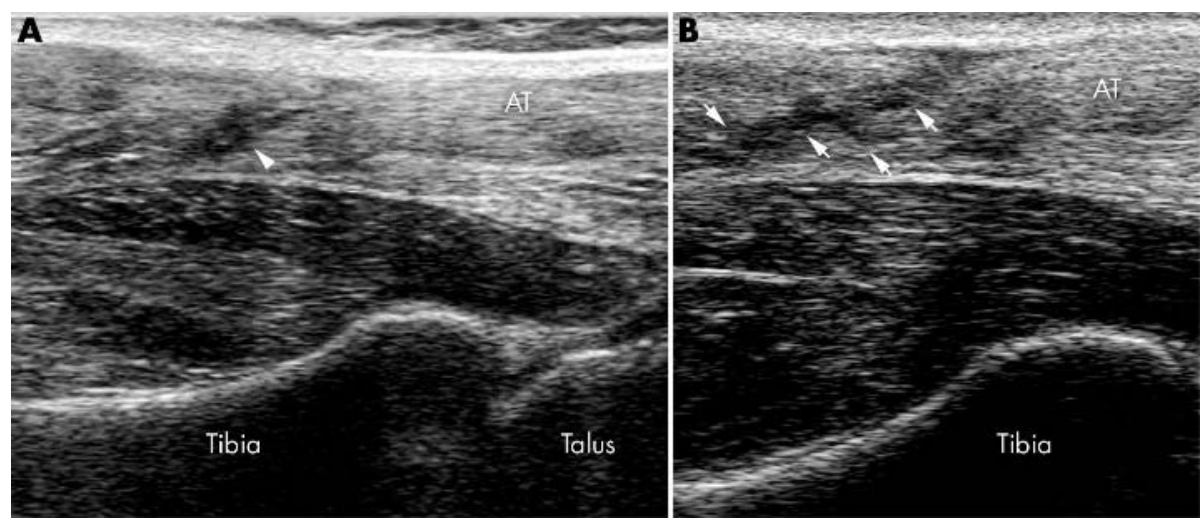

Figure 1 (A) Ultrasound image showing mainly a partial rupture in the proximal third of the Achilles tendon.

The dynamic ultrasound images show a slight but unmistakable break in tendon continuity ( $(\mathbf{)}$. Dorsal longitudinal scan obtained using a $7.5 \mathrm{MHz}$ linear transducer (Adara, Siemens). AT, Achilles tendon. (B) Same plane as in A, but scanned using a $10.0 \mathrm{MHz}$ transducer. A clear break in tendon continuity can be seen $(\uparrow)$. AT, Achilles tendon. ultrasound was also diagnosed by MRI as a partial rupture of the proximal Achilles tendon involving roughly 50\% of the tendon, which was confirmed intraoperatively. In light of the athletic ambitions of the affected patient, surgical repair was performed. The rupture zone was explored at the aforementioned site, and the tendon was debrided and sutured (fig 4). The subtotal Achilles tendon rupture was repaired with minimum invasion by means of a percutaneous, transosseous suture via the calcaneus. The foot was then immobilised in a neutral position for weeks, with subsequent functional rehabilitation.

The ultrasound follow up 12 months later showed consolidation of the tendon with still visible postoperative changes. The echo-free zone corresponding to peritendinous fluid could no longer be detected by ultrasound. The only findings were unspecific echo changes corresponding to the surgical scar. The tendon was still visibly thickened. Upon completion of treatment, all patients resumed their sports activities with no problems.

\section{DISCUSSION}

The use of ultrasound for evaluation of patients with prior tendon injuries requires a high level of operator skill. Fresh partial ruptures are detected by experienced examiners in $100 \%$ of cases. ${ }^{15}{ }^{16}$ However, this applies primarily to distal ruptures, and secondly to lateral, medial, and dorsal ruptures, that is, to those that lead to breaks in the continuity of the tendon at the typical site. Intratendinous changes in echo texture in the proximal part of the Achilles tendon have been described, ${ }^{18}$ but their clinical relevance is uncertain. Such

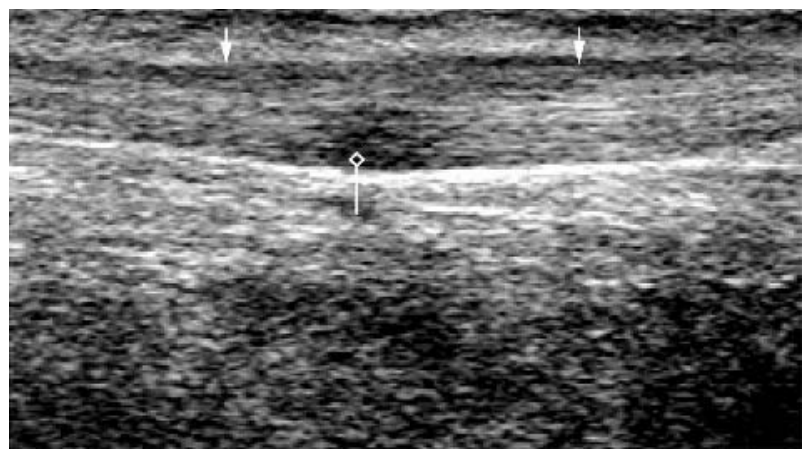

Figure 2 Ultrasound image showing an intratendinous rupture in the proximal third of the Achilles tendon; note the dorsal hypoechoic zone $(\uparrow$ ) within the tendon (open diamond above vertical line). Dorsal longitudinal scan obtained using a $7.5 \mathrm{MHz}$ linear transducer (Adara, Siemens). AT, Achilles tendon. changes were found in three of five cases in our patient population. Some authors interpret this finding as an unfavourable prognostic factor for the later development of a complete rupture of the Achilles tendon. ${ }^{19}$

Although partial rupture of the Achilles tendon is a frequent cause of chronic Achilles tendon symptoms, ${ }^{20}$ the overall occurrence of intratendinous ruptures is rare. The incidence in our patient population was also very low (1.6\%). Corticosteroid injections are associated with dual event ruptures. One of our patients with a partial rupture of the proximal Achilles tendon had had such an injection. The good sonographic detectability of these tendon injuries is likely due to the extensiveness of the changes. In particular, the fluid within the tendon could still be visualised well because the re-rupture was relatively fresh. In the case of our patient, one can assume that the rupture occurred as a result of two events, the last of which took place only a few days previously. The presence of scarring makes it much more difficult to detect these changes by ultrasound.

Epidemiological data on the incidence of intratendinous ruptures is lacking. However, when accompanied by an acute worsening of clinical symptoms, partial ruptures can be diagnosed based on their characteristic ultrasound features, especially when there is fluid surrounding and infiltrating the tendon. Sonomorphologic changes in echo texture, tendon thickening, and changes in the peritendineum are relatively unspecific signs that are associated with chronic

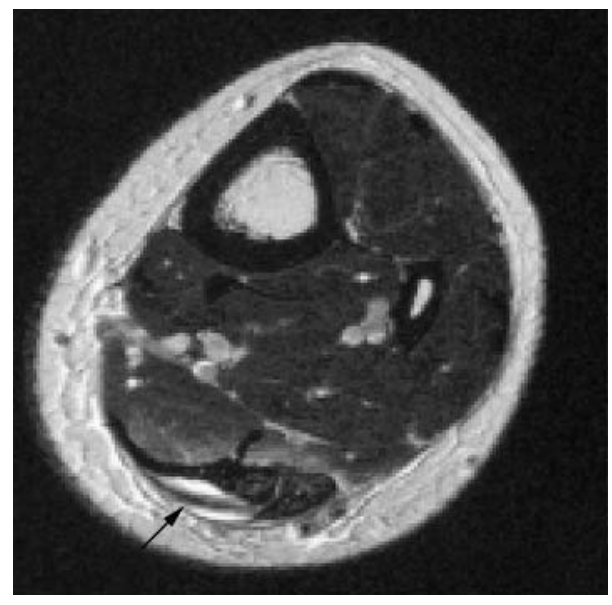

Figure 3 Corresponding T2 weighted MRI scan. Note the zone of increased signal intensity within the tendon $(\uparrow)$. 


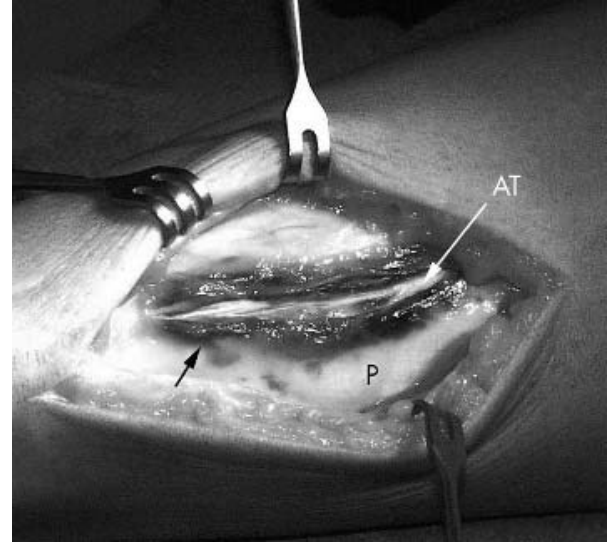

Figure 4 Intraoperative site with clearly delineated haematoma after splitting the peritendineum ( $\uparrow$ ). AT, Achilles tendon; $P$, peritendineum.

tendinopathies. The hypoechoic lesions generally observed on the lower surface of the tendon seem to be more specific. All in all, sonomorphologic parameters can, in our opinion, only suggest the diagnosis of a partial rupture of the Achilles tendon. Since the clinical features of these partial ruptures are also unspecific, one should not rely solely on the sparse findings of ultrasound alone when attempting to assess the need for surgery. MRI is able to provide an unequivocal diagnosis, especially when $\mathrm{T} 2$ weighted images enhanced by intravenous contrast medium are obtained. ${ }^{13}$ This procedure makes it possible to identify zones of increased signal intensity corresponding to zones of necrosis and fluid with a very high level of accuracy. Likewise, it is also possible to differentiate between oedema and fibrotic tissue, which is helpful in therapeutic decision making. By comparing the efficacy of MRI and ultrasound in diagnosing chronic Achilles tendon injuries, some authors ${ }^{13}$ demonstrated the superiority of MRI and others the superiority of ultrasound ${ }^{21}$ in these indications.

Because there is little experience with the sonographic assessment of such tendon disorders, magnetic resonance images should be obtained to confirm the sonographic diagnosis and determine which form of treatment is appropriate. This is especially important when treating high performance athletes as surgical treatment is more likely to be preferred.

\section{CONCLUSIONS}

When dealing with older injuries, the ultrasound findings can suggest the diagnosis of an intratendinous partial rupture of the Achilles tendon. These findings are sufficient grounds for ordering additional tests to confirm or refute the tentative sonographic diagnosis. The known advantages of ultrasound (dynamic assessment potential, wide availability, favourable time and cost factors, examination by the operator, ability to carry out follow up examinations) are important considerations. We therefore recommend ultrasound in such indications but, because of the lack of experience with ultrasound in these cases, we order a supplementary MRI evaluation for definitive presurgical diagnosis of any suspected case of intratendinous partial rupture of the proximal Achilles tendon. To what extent high frequency ultrasound transducers may be superior to conventional transducers in daily practice and whether the findings obtained using such transducers may make it possible to dispense with the need for MRI in the future must be assessed in further studies. We have had good
What is already known on this topic

Ultrasound can differentially diagnose Achilles tendinopathies and be used to assess acute Achilles tendon ruptures; an unequivocal diagnosis is usually possible in such cases. Moreover, ultrasound is now used with good success to monitor the results of treatment.

\section{What this study adds}

Ultrasound is a useful tool for evaluation of proximal Achilles tendon complaints. However, ultrasound is not sufficiently reliable for diagnosis of all pathologies, especially partial ruptures of the Achilles tendon. Thus, the definitive diagnosis must be established by MRI.

experience with high frequency transducers in cases where the Achilles tendon lies very near the surface and expect that this procedure will yield added benefits for daily routine evaluation of numerous Achilles tendinopathies.

\section{ACKNOWLEDGEMENTS}

We would like to thank Dr A Pröschild, Chief Physician at the Institute for Diagnostic Imaging, Bad Berka Central Hospital, for her critical comments and fruitful discussions and for kindly providing fig 4.

\section{Authors' affiliations}

R Kayser, C E Heyde, Charité - University Medicine, Campus Benjamin Franklin, Berlin, Germany

K Mahlfeld, Orthopedic University Clinic Magdeburg, Magdeburg, Germany

Competing interests: none declared

No benefits in any form have been or will be received from any commercial party related directly or indirectly to the subject of this article

\section{REFERENCES}

1 Segesser B, Goesele A, Renggli P. Die Achillessehne im Sport. Orthopäde 1995:24:252-67.

2 Mahlfeld K, Kayser R, Mahlfeld A, et al. Wert der Sonographie in der Diagnostik von Bursopathien im Bereich der Achillessehne. Ultraschall Med $2001 ; 22: 87-91$

3 Paavola M, Kannus P, Paakkala T, et al. Long-term prognosis of patients with Achilles tendopathy - an observational 8-year follow up study. Am J Sports Med 2000;28:634-42.

4 Kainberger F, Fialka V, Breitenseher $M$, et al. Differentialdiagnose von Erkrankungen der Achillessehne. Radiologe 1996;36:38-46

5 Majewski M, Lehmann M, Dick W, et al. Value of sonography to monitor the course of Achilles tendon rupture after treatment - comparison of conservative therapy, percutaneous tendon adaptation, and open suture. Unfallchirurg 2003; 106:556-60.

6 Rupp S, Tempelhof S, Fritsch E. Ultrasound of the Achilles tendon after surgical repair: morphology and function. Br J Radiol 1995;68:454-8

7 Kainberger F, Engel A, Tratting S, et al. Sonographische Strukturanalyse der Achillessehne und biomechanische Implikation. Ultraschall Med 1992;13:28-30.

8 Sell S, Schulz R, Balentsiefen F, et al. Lesions of the Achilles tendon - a sonographic, biomechanical and histological study. Arch Orthop Trauma Surg 1996;115:28-32.

9 Lehtinen A, Pelltokallio P, Taavitsainen M. Sonography of Achilles tendon correlated to operative findings. Ann Chir Gynaecol 1994;83:322-7.

10 Reicher MA, Basset LW, Gold RH. High-resolution magnetic resonance tomography imaging of the knee joint: normal anatomy. AJR Am J Roentgenol 1985; 145:903-9.

11 Brandser EA, El-Khoury GY, Saltzmann CL. Tendon injuries: application of magnetic resonance tomography. Can Assoc Radiol J 1995;46:9-18.

12 Jacobson JA. Musculoskeletal sonography and MR imaging. A role for both imaging methods. Radiol Clin North Am 1999;37:713-35.

13 Movin T, Kristoffersen-Wiberg M, Rolf C, et al. MR imaging in chronic Achilles tendon disorders. Acta Radiol 1998;39:126-32. 
14 Movin T, Kristoffersen-Wiberg M, Shalabi A, et al. Intratendinous alteration as imaged by ultrasound and contrast medium enhanced magnetic resonance in chronic achillodynia. Foot Ankle Int 1998;19:311-17

15 Kalebo P, Allenmark C, Peterson L, et al. Diagnostic value of ultrasonography in partial ruptures of the Achilles tendon. Am J Sports Med 1992;20:378-81.

16 Kalebo P, Goksor LA, Sward L, et al. Soft-tissue radiography, computed tomography, and ultrasonography of partial Achilles tendon ruptures. Acta Radiol 1990;31:565-70.

17 Merk H. Die hochauflösende Real-Time-Sonographie in der Diagnostik von Achillessehnenerkrankungen. Ultraschall Med, 1989;10:192-7.

18 Gibbon WW, Cooper JR, Radcliffe GS. Sonographic incidence of tendon microtears in athletes with chronic Achilles tendinosis. Br J Sports Med 1999;33:129-30.

19 Nehrer S, Breitseher M, Brodner W, et al. Clinical and sonographic evaluation of risk of rupture in the Achilles tendon. Arch Orthop Trauma Surg 1997; 116:14-18.

20 Williams JPG. Achilles tendon lesions in sports. Sports Med 1986:3:114-35.

21 Khan KM, Forster BB, Robinson J, et al. Are ultrasound and magnetic resonance imaging of value in assessment of Achilles tendon disorders? A two year prospective study. Br J Sports Med 2003;37:149-53.

\section{............ COMMENTARY}

The material presented highlights a clinical problem that is frequently encountered in athletes. Ultrasonography seems to be a valid screening method for Achilles tendon pathologies: it provides the advantage of "dynamic" imaging and thus may visualise the clinical findings, especially if the clinician (orthopaedic surgeon) is personally performing the ultrasound assessment. However, the method may have limitations, so the authors are cautious and recommend preoperative verification by MRI after sonographic screening in cases that are scheduled for surgery.

C Tschauner Central Hospital Bozen, Bozen, Italy; christian.tschauner@sbbz.it 